\title{
Estimación de Edad Dentaria Utilizando el Método de Demirjian en Niños de 5 a 15 Años de Curicó, Chile
}

\author{
Dental Age Estimation Using the Demirjian's Method \\ in Children 5 to 15 Years of Age in Curicó, Chile
}

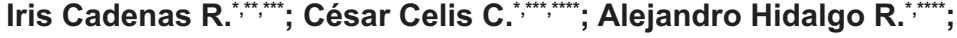 \\ Alejandro Schilling Q., ${ }^{* * * * * * * *}$ \& Jaime San Pedro V., ${ }^{* * *+*}$
}

\begin{abstract}
CADENAS, R. I.; CELIS, C. C.; HIDALGO, R. A.; SCHILLING Q. A. \& SAN PEDRO, V. J. Estimación de edad dentaria utilizando el método de Demirjian en niños de 5 a 15 años de Curicó, Chile. Int. J. Odontostomat., 8(3):453-459, 2014.

RESUMEN: El método de Demirjian (MD) es el más ampliamente difundido para estimar la edad dental (ED). Con este método se han realizado numerosos estudios en diferentes grupos étnicos, analizándose niños europeos, asiáticos y norteamericanos entre otros. Estos resultados sugieren posibles diferencias en los patrones de maduración dental entre las diferentes poblaciones. El objetivo del presente estudio fue determinar la edad dentaria aplicando el MD en una muestra de 363 ortopantomografías de pacientes entre 5 y 15 años, obtenidas de un centro radiológico maxilofacial privado en Curicó, VII región, Chile. Se obtuvo un coeficiente de correlación inter-clase $(\mathrm{CCl})$, como medida de correlación entre edad dental y cronológica, de 0,974 (CCI 95\% 0,968-0,979). El CCI para niños fue de 0,972 (CCI 95\% 0,963-0,979) y en el caso de las niñas fue de 0,977 (CCI 95\% 0,969-0,983). En general, el método de Demirjian tendió a sobreestimar la edad en los niños y niñas. La ED observada fue mayor que la edad cronológica (EC) en 130 niños y en 138 niñas, resultó igual a la EC en 13 niños y 8 niñas, y fue menor que la EC en 38 niños y 33 niñas. En conclusión, nuestros resultados coinciden con hallazgos en numerosos lugares del mundo, es decir, la ED en este grupo se fue levemente mayor que la muestra original francocanadiense. Sin embargo, el rango obtenido de la ED es similar al de la EC y el grado de correlación entre ambas es casi perfecto al aplicar el CCl. El grado de correlación entre ambas edades para cada sexo también es muy bueno. Por lo tanto, consideramos que el MD es aplicable a esta muestra de niños.
\end{abstract}

PALABRAS CLAVE: edad dental, estadios de desarrollo dentario, método de Demirjian.

\section{INTRODUCCIÓN}

La estimación de la edad constituye una parte importante del proceso rutinario de identificación de cadáveres y es fundamental en la investigación de crímenes y accidentes. La edad cronológica (EC) es importante en la mayoría de las sociedades en términos de escolarización, empleo y matrimonio (Willems, 2001). En el área forense el diagnóstico de la edad debe comprender un examen físico, la evaluación de signos de maduración sexual, desórdenes del desarrollo, examen radiográfico de la mano izquierda, examen dentario y una ortopantomografía (OPG) (Martin-de las Heras et al., 2008). La estimación de la edad dental (ED) en personas vivas se basa principalmente en métodos no invasivos, y es generalmente aceptada como el método más confiable para estimar la edad cronológica en niños (Meinl, 2007; Olze et al., 2006) debido a su baja variabilidad (Willems). Los dientes en desarrollo se ven menos afectados que otros tejidos corporales por endocrinopatías o por agresiones ambientales, como mala nutrición u otras alteraciones patológicas (Meinl; Olze et al.). El proceso de maduración dental se correlaciona con diferentes estadios de mineralización que pueden ser observados a través de registros radiográficos (Prieto, 2008). Estos estadios constituyen formas dentarias fácilmente reconocibles, desde el inicio de la calcificación hasta su forma madura final (Demirjian et al., 1973).

Especialista en Radiología Maxilofacial, Talca, Chile.

* Unidad de Radiología Maxilofacial, Servicio de Odontología, Hospital de Curicó, Curicó, Chile.

*** Macef Centro Radiológico, Curicó, Chile.

*n** Servicio de Radiología Maxilofacial, Departamento de Estomatología, Facultad de Ciencias de la Salud, Universidad de Talca, Talca, Chile. 
En 1973, Demirjian et al. presentaron un trabajo de una población Franco-canadiense en que describieron 8 estadios distintivos, denominados estadios A-H, definidos por cambios morfológicos y que no dependen de estimaciones especulativas de longitud. Aplicaron una escala de maduración basada en el método de Tanner et al. (1975) para estimar edad cronológica, obteniéndose un puntaje para cada estadio por diente, separadamente para niños y niñas. Se evalúan todos los dientes permanentes mandibulares izquierdos (excluyendo el tercer molar). La suma de los puntajes de los 7 dientes entrega un valor de madurez dentaria en escala de 0 a 100, la cual puede ser convertida directamente en edad dentaria utilizando las tablas y curvas de percentiles que los autores entregan.

El método de Demirjian (MD) es ampliamente difundido para estimar la ED. Se han realizado numerosos estudios en diferentes grupos étnicos, analizándose niños europeos, asiáticos y sudamericanos, entre otros. Se ha sugerido que puede haber diferentes patrones de maduración dental entre las distintas poblaciones, no sólo entre grupos poblacionales alrededor de todo el mundo, sino también entre individuos de diferentes ciudades o áreas geográficas dentro del mismo país (Cruz-Landeira et al., 2010; Eid et al., 2002; Lee et al., 2008; Liversidge et al., 1999; Moananui et al., 2008; Nyström et al., 2000; Nyström et al., 1988; Flores, et al., 2010; Teivens \& Mörnstad, 2001).

El objetivo de este estudio fue estimar la ED aplicando el MD en niños entre los 5 y los 15 años en la Región del Maule, Chile.

\section{MATERIAL y MÉTODO}

La muestra se obtuvo desde el archivo digital de una clínica de radiología maxilofacial de Curicó, Región del Maule. Comprendió 363 OPG con un rango de edad entre 5 y 15 años atendidos entre el año 2009 y 2010. La distribución por sexo y edad se observa en la Figura 1.

Se utilizó un ortopantomógrafo digital Sirona Ortophos XG 5 (Sirona, Siemens, Alemania). Las radiografías fueron archivadas en formato JPEG (2440 x 1292 píxeles) y analizadas en el computador del observador (IC). Se excluyeron las imágenes que presentaran un grado de distorsión que dificultara la visualización de dientes en la zona a examinar, aquellas que presentaran patologías como quistes o tumores, radiografías de pacientes en tratamiento de ortodoncia y aquellas que presentaran ausencia de dientes debido a agenesia o a exodoncia.

Previo a la evaluación de la muestra final, se seleccionaron al azar 51 OPG para calcular la variabilidad intra-evaluador, las que fueron evaluadas por un solo observador (IC) dos veces con espacio de 3 semanas entre la primera y la segunda evaluación, obteniéndose un índice kappa de 0,8.

Los estadios de desarrollo de los siete dientes mandibulares se analizaron según el método propuesto por Demirjian et al., en el siguiente orden: incisivo central, incisivo lateral, canino, primer premolar, segundo premolar, primer molar y segundo molar denominán-

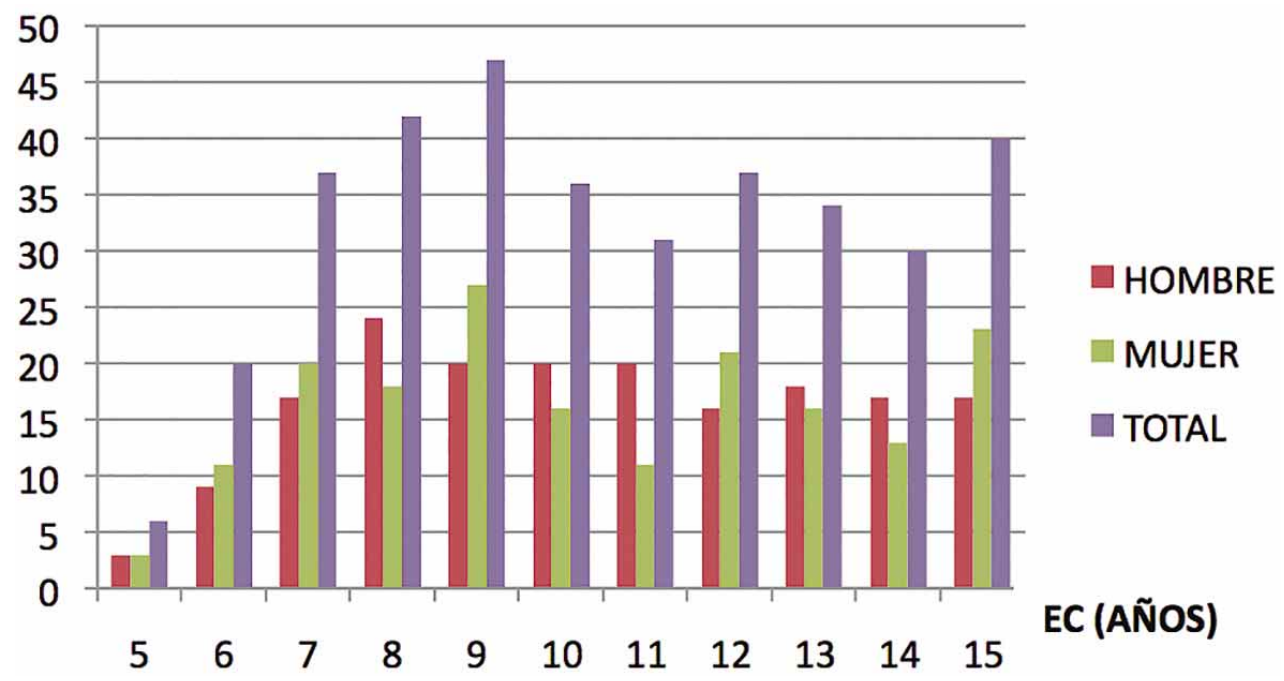

Fig. 1. Gráfico distribución de edad cronológica (EC) por sexo y por grupo etario. 
dose cada como IC, IL, C, 1PM, 2PM, 1M Y 2M, respectivamente.

Se confeccionó una hoja de trabajo en Microsoft Office Excel 2007 para ingreso de los pacientes, asignándosele a cada caso un número $(1,2,3,4)$ y realizar cálculos de puntajes y edad dentaria. El observador no tuvo como antecedente la fecha de nacimiento de cada sujeto al momento de la evaluación. Posteriormente los casos se separaron en dos planillas diferentes, una para los niños y otra para las niñas.

Los estadios se transformaron de letras a números (de A, B, C, D, E, F, G, H a 1, 2, 3, 4, 5, 6, 7, 8, respectivamente) (Tabla I) para facilitar la asignación del puntaje de maduración correspondiente a cada diente según las tablas del método original.

Tabla I. Conversión de Estadíos de Demirjian.

\begin{tabular}{cc}
\hline Estadio & Conversión \\
\hline A & 1 \\
B & 2 \\
C & 3 \\
D & 4 \\
E & 5 \\
F & 6 \\
G & 7 \\
H & 8 \\
\hline
\end{tabular}

Los puntajes fueron asignados siguiendo cuidadosamente los criterios descritos para cada estadio dentario, y comparando los dientes con los diagramas e imágenes radiográficas entregadas por Demirjian et al. En caso de dudas entre dos estadios, se asignó al diente el estadio de menor desarrollo. Posteriormente se obtuvo el porcentaje de maduración y la edad dentaria con un decimal. La edad cronológica (EC) se calculó con un decimal, sustrayendo la fecha de nacimiento y la fecha de toma de la OPG.

Análisis. Los resultados se ingresaron al software SPSS para Windows versión 17.0 (SPSS Inc., Chicago, IL), el que se utilizó para todos los análisis. Se calculó el coeficiente de correlación inter-clase $(\mathrm{CCl})$ para medir la correspondencia entre EC y ED. Se utilizó un gráfico Bland-Altman para ilustrar la correlación.
Las diferencias entre EC y ED estimada fueron testeadas estadísticamente usando las pruebas t pareada y de Wilcoxon; se consideró un valor de $p<0,05$ y $p<0,01$, respectivamente, como estadísticamente significativo. Como el tamaño de la muestra era menor de 30 y presentaba una distribución no normal en algunos grupos, estaba indicado el uso de pruebas no paramétricas (Wilcoxon signed rank test). Para ser consistente en todos los grupos, sin embargo, se aplicaron tanto pruebas paramétricas como no paramétricas.

\section{RESULTADOS}

El ICC se puede observar para niños, niñas y en general, en la Tabla II. El gráfico Bland-Altman ilustra que la muestra se concentró dentro de los límites de confianza definidos para cada muestra, que fue $\pm 1,6$ en todos los casos (niños, niñas y para el total) (Figs. 2, 3 y 4 )

Tabla II Coeficiente de Correlación Intra clase, $\mathrm{p}<$ 0,05 .

\begin{tabular}{lcc}
\hline & ICC & IC 95\% \\
\hline Niños & 0,972 & $0,963-0,979$ \\
Niñas & 0,977 & $0,969-0,983$ \\
Total & 0,974 & $0,968-0,979$ \\
\hline
\end{tabular}

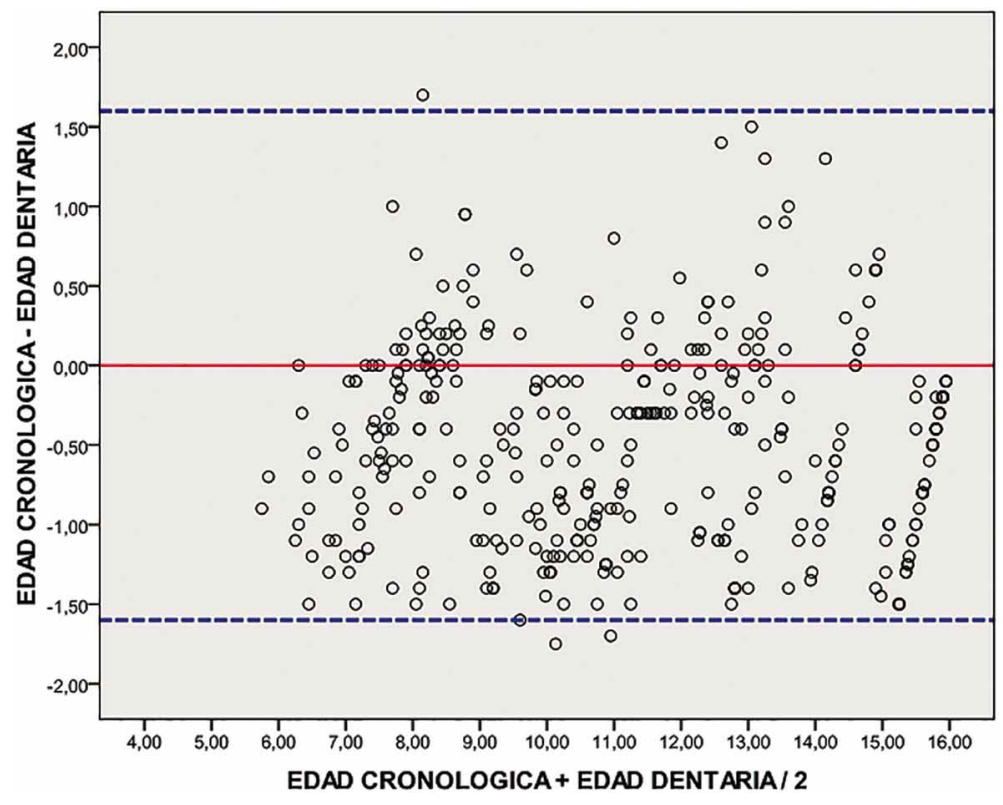

Fig. 2. Gráfico Bland-Altman para niños y niñas. 


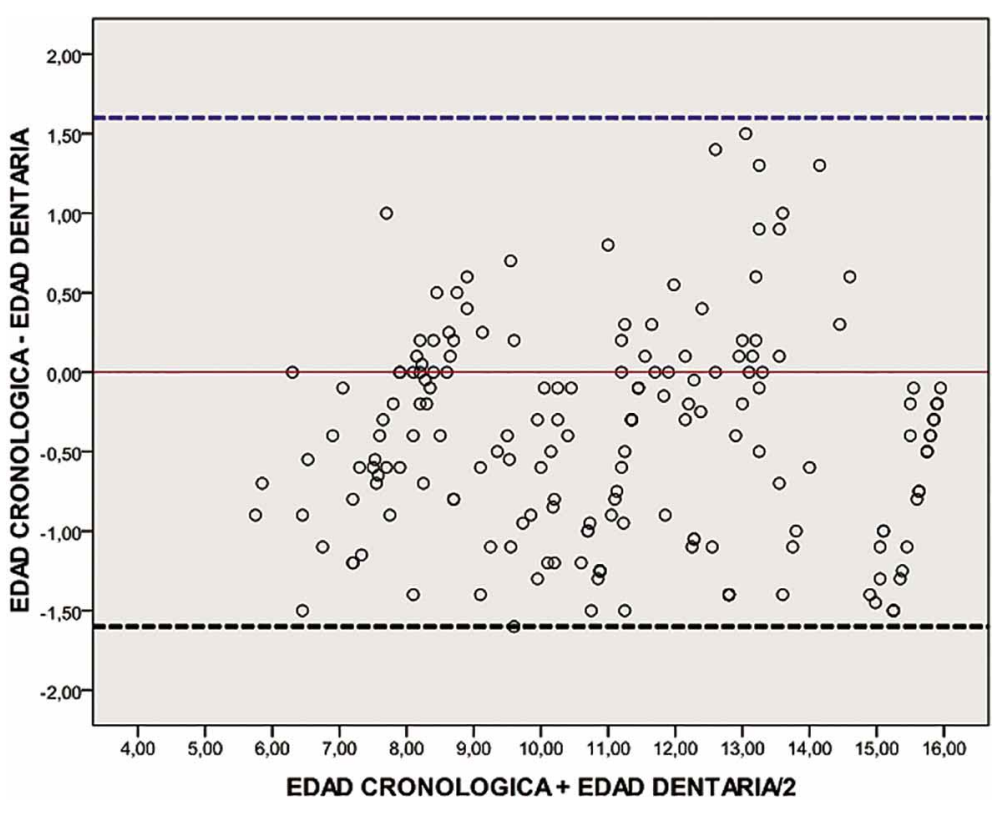

Fig. 3. Gráfico Bland-Altman para niños.

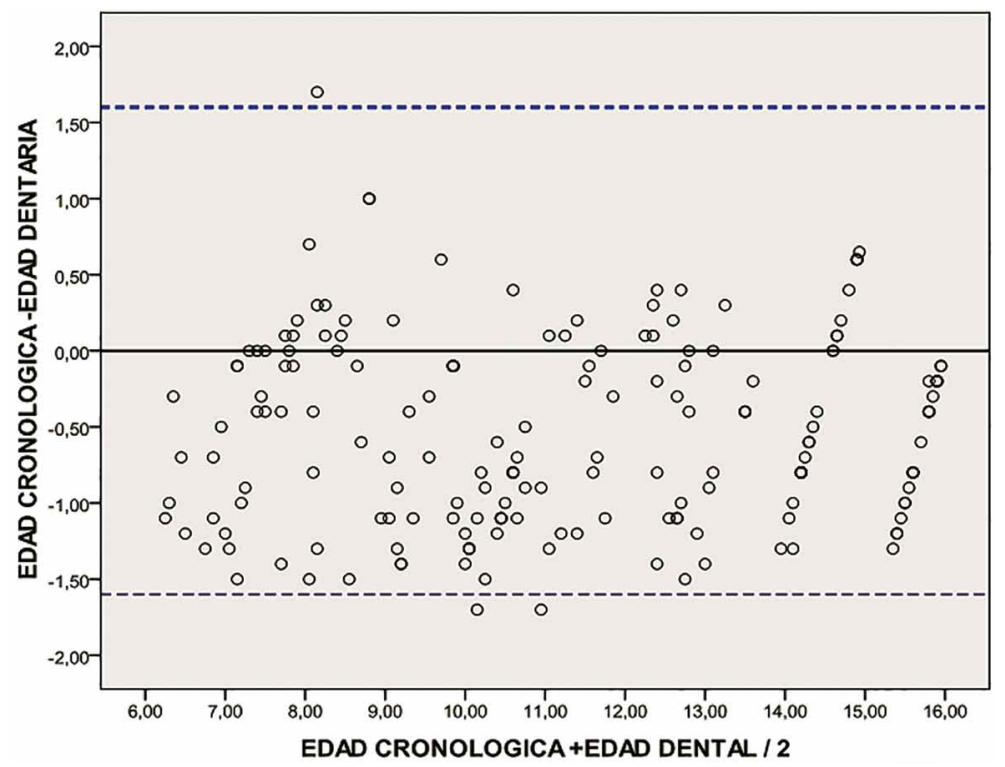

Fig. 4 Gráfico Bland-Altman para niñas.

Los rangos de edades dentarias y edades cronológicas para ambos sexos se exponen en la Tabla III.

Tabla III. Edad Cronológica versus Edad Dentaria para niños y niñas.

\begin{tabular}{lcc}
\hline & EC & ED \\
\hline Niños & $5,3-15,9$ & $6,2-16$ \\
Niñas & $5,7-15,9$ & $6,5-16$ \\
Total & $5,3-15,9$ & $6,2-16$ \\
\hline
\end{tabular}

Al aplicar la prueba t se encontró diferencia estadísticamente significativa entre EC y ED para todos los grupos etarios y en el total en las niñas, mientras que al aplicar la prueba de Wilcoxon la diferencia resultó significativa sólo en los grupos de $13,12,10,9,7$ y 6 años de edad (Tabla IV). En los niños, se encontraron diferencias estadísticamente significativas en los grupos etarios de $6,7,9,10,12,14$ y 15 , y en el total en los niños, mientras que al aplicar la prueba de Wilcoxon la diferencia fue significativa sólo en los grupos de 15,10, 7 y años de edad y en el total (Tabla V). Para las niñas, la diferencia de medias entre EC y ED para el total (179) fue de -0,54 (DE $0,63)$ años, resultando la mayor diferencia de medias en el grupo etario de 5 años, obteniéndose -1,1 (DE 0,1) años y la menor en el grupo de 15 años, que resultó ser de $-0,25$ (DE 0,53) años (Tabla IV).

Para los hombres, la diferencia de medias entre EC y ED para el total (181) fue de -0,42 (DE 0,66) años, encontrándose que la mayor diferencia de medias se presentó en el grupo etario de 13 años, correspondiendo a 1,33 (DE 0,77) años y la menor en el grupo de 8 años, que fue de 0,19 (DE 0,6) años (Tabla V).

En general, el método de Demirjian tendió a sobreestimar la edad en los niños y niñas de esta muestra. La ED observada fue mayor que la EC en 130 niños y en 138 niñas. La ED observada fue igual a la EC en 13 niños y 8 niñas, mientras que la ED fue menor que la EC en 38 niños y 33 niñas (Tabla VI).

\section{DISCUSIÓN}

En el transcurso de los años, muchos investigadores han analizado la precisión del MD para diferentes poblaciones en los cinco continentes (Al-Emran, 2008; Cadenas et al., 2010; Chen et al., 2010; Chertkow \& Fatti, 1979; Cruz-Landeira, et al.; Davidson \& Rodd, 2001; Eid et al.; Kanbur et al., 2006; Koshy \& Tandon, 1998; Krailassiri et al., 2002; Leurs et al., 2005; 
Tabla IV. Test-t y Wilcoxon Niñas.

\begin{tabular}{|c|c|c|c|c|c|c|c|c|c|}
\hline EC & $n$ & $\begin{array}{l}\text { Media EC } \\
\quad \pm D E\end{array}$ & $\begin{array}{l}\text { Media ED } \\
\pm D E\end{array}$ & $\begin{array}{l}\text { Media DIF } \\
\pm \mathrm{DE}\end{array}$ & IC 95\% & $t$ & df & $\begin{array}{l}\text { T sig. } \\
p<0,05\end{array}$ & $\begin{array}{l}\text { Wilcoxon } \\
p<0,01\end{array}$ \\
\hline 15 & 17 & $15,44 \pm 0,41$ & $15,69 \pm 0,68$ & $-0,25 \pm 0,53$ & $-0,48 \pm 0,02$ & $-2,24$ & 22 & ${ }^{*} 0,036$ & 0,041 \\
\hline 14 & 13 & $14,53 \pm 0,33$ & $15,03 \pm 0,67$ & $-0,5 \pm 0,56$ & $-0,84 \pm 0,16$ & $-3,23$ & 12 & ${ }^{*} 0,007$ & 0,016 \\
\hline 13 & 16 & $13,52 \pm 0,25$ & $14,18 \pm 0,58$ & $-0,67 \pm 0,45$ & $-0,9 \pm 0,43$ & $-5,91$ & 15 & ${ }^{*} 0,00$ & ${ }^{*} 0,001$ \\
\hline 12 & 21 & $12,4 \pm 0,28$ & $12,9 \pm 0,49$ & $-0,5 \pm 0,63$ & $-0,78 \pm 0,21$ & $-3,62$ & 20 & ${ }^{*} 0,002$ & ${ }^{*} 0,008$ \\
\hline 11 & 11 & $11,4 \pm 0,22$ & $11,8 \pm 0,58$ & $-0,27 \pm 0,9$ & $-0,75 \pm 0,03$ & $-2,41$ & 10 & ${ }^{\star} 0,036$ & 0,047 \\
\hline 10 & 16 & $10,32 \pm 0,26$ & $11,1 \pm 0,62$ & $-0,78 \pm 0,6$ & $-1,09 \pm 0,47$ & $-5,4$ & 15 & ${ }^{*} 0,00$ & ${ }^{*} 0,001$ \\
\hline 9 & 27 & $9,49 \pm 0,28$ & $10,14 \pm 0,96$ & $-0,64 \pm 0,84$ & $-0,97 \pm 0,31$ & $-3,9$ & 26 & ${ }^{*} 0,00$ & ${ }^{*} 0,001$ \\
\hline 8 & 18 & $8,46 \pm 0,18$ & $8,9 \pm 0,79$ & $-0,44 \pm 0,7$ & $-0,78 \pm 0,09$ & $-2,7$ & 17 & ${ }^{*} 0,015$ & 0,049 \\
\hline 7 & 20 & $7,48 \pm 0,29$ & $7,93 \pm 0,58$ & $-0,44 \pm 0,55$ & $-0,69 \pm 0,18$ & $-3,56$ & 19 & ${ }^{*} 0,002$ & ${ }^{*} 0,001$ \\
\hline 6 & 11 & $6,42 \pm 0,24$ & $7,37 \pm 0,42$ & $-0,95 \pm 0,37$ & $-1,2 \pm 0,7$ & $-8,5$ & 10 & ${ }^{*} 0,00$ & ${ }^{*} 0,003$ \\
\hline 5 & 3 & $5,8 \pm 0,1$ & $6,9 \pm 0,17$ & $-1,1 \pm 0,1$ & $-1,35 \pm 0,85$ & $-19,05$ & 2 & ${ }^{\star} 0,003$ & 0,109 \\
\hline TO & 179 & $10,94 \pm 2,92$ & $11,48 \pm 2,88$ & $-0,54 \pm 0,63$ & $-0,63 \pm 0,45$ & $-11,58$ & 178 & ${ }^{*} 0,00$ & ${ }^{*} 0,00$ \\
\hline
\end{tabular}

Tabla V. Test-t y Wilcoxon Niños.

\begin{tabular}{|c|c|c|c|c|c|c|c|c|c|}
\hline EC & $\mathbf{n}$ & $\begin{array}{l}\text { Media EC } \\
\quad \pm \mathrm{DE}\end{array}$ & $\begin{array}{l}\text { Media ED } \\
\pm \mathrm{DE}\end{array}$ & $\begin{array}{l}\text { Media DIF } \pm \\
\text { DE }\end{array}$ & IC $95 \%$ dif & $\mathbf{t}$ & df & $\begin{array}{c}\text { Sig. } \\
p<0,05\end{array}$ & $\begin{array}{c}\text { Wilcoxon } \\
p<0,01\end{array}$ \\
\hline 15 & 17 & $15,55 \pm 0,22$ & $15,94 \pm 0,15$ & $-0,39 \pm 0,22$ & $-0,5 \pm 0,27$ & $-7,192$ & 16 & $0^{*}$ & ${ }^{*} 0,00$ \\
\hline 14 & 17 & $14,52 \pm 0,26$ & $15,18 \pm 1,06$ & $-0,66 \pm 1,02$ & $-0,9 \pm 0,14$ & $-2,69$ & 16 & $0,016^{*}$ & 0,01 \\
\hline 13 & 18 & $13,35 \pm 0,28$ & $13,22 \pm 0,67$ & $1,33 \pm 0,77$ & $-0,25 \pm 0,52$ & 0,731 & 17 & 0,475 & 0,44 \\
\hline 12 & 16 & $12,32 \pm 0,31$ & $12,85 \pm 0,7$ & $-0,53 \pm 0,7$ & $-0,9 \pm 1,5$ & $-3,005$ & 15 & $0,009^{*}$ & 0,018 \\
\hline 11 & 20 & $11,47 \pm 0,25$ & $11,68 \pm 0,59$ & $-0,21 \pm 0,5$ & $0,1 \pm 0,44$ & $-1,898$ & 19 & 0,073 & 0,8 \\
\hline 10 & 20 & $10,33 \pm 0,27$ & $11,19 \pm 0,52$ & $-0,86 \pm 0,46$ & $-1,07 \pm 6,46$ & $-8,4$ & 19 & $0^{*}$ & ${ }^{*} 0,00$ \\
\hline 9 & 20 & $9,44 \pm 0,3$ & $9,86 \pm 0,74$ & $-0,43 \pm 0,65$ & $-0,73 \pm 0,12$ & $-2,926$ & 19 & $0,009^{*}$ & 0,011 \\
\hline 8 & 24 & $8,42 \pm 0,24$ & $8,61 \pm 0,69$ & $-0,19 \pm 0,6$ & $-0,44 \pm 0,06$ & $-1,59$ & 23 & 0,125 & 0,254 \\
\hline 7 & 17 & $7,46 \pm 0,31$ & $7,97 \pm 0,38$ & $-0,51 \pm 0,35$ & $-0,69 \pm 0,33$ & $-6,087$ & 16 & $0^{*}$ & ${ }^{*} 0,001$ \\
\hline 6 & 9 & $6,47 \pm 0,28$ & $7,28 \pm 0,55$ & $-0,81 \pm 0,42$ & $-1,13 \pm 0,49$ & $-5,8$ & 8 & $0^{*}$ & 0,012 \\
\hline 5 & 3 & $5,5 \pm 0,2$ & $6,53 \pm 0,57$ & $-1,03 \pm 0,42$ & $-2,07 \pm 0,001$ & $-4,3$ & 2 & 0,5 & 0,109 \\
\hline TO & 18 & $10,93 \pm 2,8$ & $11,35 \pm 2,8$ & $-0,43 \pm 0,66$ & $0,52 \pm 0,33$ & $-8,6$ & 180 & $0^{*}$ & ${ }^{*} 0$ \\
\hline
\end{tabular}

Tabla VI. Wilcoxon signedranked test, $p<0,01$.

\begin{tabular}{ccc}
\hline & Niños & Niñas \\
\hline$E D>E C$ & 130 & 138 \\
$E D=E C$ & 13 & 8 \\
ED<EC & 38 & 33 \\
Total & 181 & 179 \\
\hline
\end{tabular}

Maia et al., 2010; McKenna et al., 2002; Mitchell et al., 2009; Nadler, 1998; Nyström et al., 2000; Tunc \& Koyuturk, 2008; Uysal et al., 2004), obteniendo en general una sobreestimación de la edad dentaria.

Hay pocos datos sobre la aplicación del MD en Centro y Sudamérica. En un estudio del sureste de Brasil, se obtuvo una sobreestimación de la EC de 0,68 (DE 0,23) años en los niños y de 0,62 (DE 0,20) años en las niñas (Eid et al.) En otro estudio al noreste del mismo país, se obtuvo una sobreestimación de la EC de 1,22 años en los niños y de 1,3 años en las niñas (Maia et al.).

Recientemente, se evaluó la madurez dentaria obtenida utilizando el MD en niños españoles y una muestra de niños venezolanos amerindios, obteniéndose una sobreestimación de la EC de 0,76 (DE 1,01) años $y$ de 0,88 (DE 1,09) años en los niños y niñas españoles, respectivamente, mientras que en el grupo venezolano, la EC fue subestimada en 0,23 (DE 0,93) años en los niños y 0,1 (DE 1,04) años en las niñas. Esto último no concuerda con la tendencia general. Los autores sugieren que dicho resultado, si bien puede deberse a un tamaño muestral reducido, también se podría atribuir en parte a la etnia (Cruz-Landeira et al.). En el presente estudio, la sobreestimación de la EC resultó ser levemente menor que la observada en los trabajos antes mencionados, realizados en América del Sur. 
En relación a la población chilena, sólo se ha publicado un estudio de correlación, en el que se aplicó el MD en un grupo de 159 niños y niñas de entre 3 y 14 años de edad en la ciudad de Concepción. La edad dental observada para los diversos grupos etarios fluctuó entre los 3,2 y 14,2 años. El coeficiente de correlación intra-clase, como medida de correspondencia entre ED y EC fue de 0,98 (IC 95\%: 0,97-0,99). EI $\mathrm{CCl}$ fue de 0,97 (IC 95\%: 0,96-0,98) para los niños y 0.98 (IC 95\%: 0,97-0,99) para las niñas, por lo que los autores concluyen que el método es aplicable en nuestra población (Pérez et al.). Dichos resultados son muy similares a los obtenidos en el presente estudio (ICC para niños y niñas = 0,97 (IC 95\% 0,968-0,979), para niños $=0,97$ (IC 95\% 0,963-0,979) y para niñas = 0,97 (IC 95\% 0,969-0,983) (Tabla II). Sin embargo, por tratarse de un estudio de correlación, no entrega información respecto de posibles diferencias entre EC y ED.

El método de Demirjian fue aplicable a la muestra de niños del presente estudio. Sin embargo, los niños chilenos presentaron una ED significativamente mayor que la EC, es decir, la maduración dentaria en este grupo se encuentra avanzada en comparación con la muestra original franco-canadiense. A pesar de que esto coincide con hallazgos en numerosos luga- res del mundo, y que la mayoría de los autores sugiere la necesidad de aplicar estándares propios, se debe considerar la hipótesis del cambio secular y el hecho de que se ha demostrado que la cronología de la formación dentaria no difiere significativamente entre las diferentes poblaciones, de modo que la aplicación de las adaptaciones previamente mencionadas podría no ser necesaria. Al igual que numerosos estudios, el presente utiliza una muestra muy pequeña, dado que corresponde a un estudio preliminar por lo que las diferencias entre ED y EC obtenidas de esta manera pueden tener muy poco o ningún significado biológico (Liversidge, 2010; Reid \& Dean, 2006). Se requiere nuevos estudios que utilicen muestras más numerosas y que, idealmente, incluyan niños de edades menores a las que se utilizaron en el presente. Resultaría interesante, además, poder aplicar el MD en grupos de niños de otras áreas geográficas de Chile, ya que permitiría realizar comparaciones y evaluar posibles diferencias entre grupos en un mismo país.

En conclusión, el rango de la edad cronológica es similar al de edad dentaria y el grado de correlación entre las edades cronológica y dental es casi perfecto al aplicar el $\mathrm{CCl}$. Se encontró un grado de correlación muy bueno entre las edades cronológica y dentaria para cada sexo.

CADENAS, R. I.; CELIS, C. C.; HIDALGO, R. A.; SCHILLING Q. A. \& SAN PEDRO, V. J. Dental age estimation using the Demirjian's method in children 5 to 15 years of age in Curicó, Chile. Int. J. Odontostomat., 8(3):447-452, 2014.

ABSTRACT: The Demirjian's method (DM) is the most widely used method for estimating dental age. A number of studies with this method have been performed in different ethnic groups, with European, Asian y North American children being analyzed among others. Possible differences in patterns of dental maturation among different population have been suggested. The aim of this study was to determine dental age with the DM in a sample of 363 panoramic radiographs from children 5-15 years old, obtained from a private radiological maxillofacial center in Curicó, Chile. An inter-class correlation coefficient (ICC) was obtained as a measure of correlation between dental and chronological age of 0.974 (ICC 95\% 0.968-0.979). The ICC for boys was 0.972 (ICC 95\% 0.963- 0.979) and 0.977 for girls (CCI 95\% 0.969-0.983). In general, DM overestimated the age in girls and boys. Dental age (DA) was observed to be higher than chronological age (CA) in 130 boys and 138 girls; DA was equal to CA in 13 boys y 8 girls and lower than CE in 38 boys and 33 girls. In conclusion, our study concurs with several other studies in other places of the world, indeed the DA was found to be higher when compared to the original French-Canadian sample. However, the range obtained for DA is similar to CA and the degree of correlation between both is almost perfect when applying ICC. The degree of correlation between both ages for each sex was found as very good. Furthermore, we consider that DM can be applied to this sample of children.

KEY WORDS: dental age, stages of dental development, Demirjian's method.

\section{REFERENCIAS BIBLIOGRÁFICAS}

Al-Emran, S. Dental age assessment of 8.5 to 17 Year-old Saudi children using Demirjian's method. J. Contemp. Dent. Pract., 9(3):64-71, 2008.

Cadenas, I.; Celis, C. \& Hidalgo, A. Método de Demirjian para estimación de edad dentaria en base a estadios de mineralización. Anu. Soc. Radiol. Oral Máxilo. Fac. Chil., 13:17-23, 2010.

Chen, J. W.; Guo, J.; Zhou, J.; Liu, R. K.; Chen, T. T. \& Zou, S. J. 458
Assessment of dental maturity of western Chinese children using Demirjian's method. Forensic Sci. Int., 197(1-3):119.e14, 2010 .

Chertkow, S. \& Fatti, P. The relationship between tooth mineralization and early radiographic evidence of the ulnar sesamoid. Angle Orthod., 49(4):282-8, 1979.

Cruz-Landeira, A.; Linares-Argote, J.; Martínez-Rodríguez, M.; Rodríguez-Calvo, M. S.; Otero, X. L. \& Concheiro, L. Dental age estimation in Spanish and Venezuelan children. Comparison 
of Demirjian and Chaillet's scores. Int. J. Legal Med., 124(2):10512,2010

Davidson, L. E. \& Rodd, H. D. Interrelationship between dental age and chronological age in Somali children. Community Dent Health, 18(1):27-30, 2001.

Demirjian, A.; Goldstein, H. \& Tanner, J. M. A new system of dental age assessment. Hum. Biol, 45(2):211-7, 1973.

Eid, R. M.; Simi, R.; Friggi, M. N. \& Fisberg, M. Assessment of dental maturity of Brazilian children aged 6 to 14 years using Demirjian's method. Int. J. Paediatr. Dent., 12(6):423-8, 2002.

Flores, A. P.; Sanhueza, M. A.; Barboza, P. \& Monti, C. F. Study of Chilean children's dental maturation. J. Forensic Sci., 55(3):7357, 2010.

Kanbur, N. O.; Kanli, A.; Derman, O.; Eifan, A. \& Ataç, A. The relationships between dental age, chronological age and bone age in Turkish adolescents with constitutional delay of growth. J. Pediatr. Endocrinol. Metab., 19(8):979-85, 2006.

Koshy, S. \& Tandon, S. Dental age assessment: the applicability of Demirjian's method in south Indian children. Forensic Sci. Int., 94(1-2):73-85, 1998.

Krailassiri, S.; Anuwongnukroh, N. \& Dechkunakorn, S. Relationships between dental calcification stages and skeletal maturity indicators in Thai individuals. Angle Orhtod., 72(2):155-66, 2002.

Lee, S. E.; Lee, S. H.; Lee, J. Y.; Park, H. K. \& Kim, Y. K. Age estimation of Korean children based on dental maturity. Forensic Sci. Int., 178(2-3):125-31, 2008.

Leurs, I. H.; Wattel, E.; Aartman, I. H.; Etty, E. \& Prahl-Andersen, B. Dental age in Dutch children. Eur. J. Orthod., 27(3):309-14, 2005.

Liversidge, H. M. Interpreting group differences using Demirjian's dental maturity method. Forensic Sci. Int., 201(1-3):95-101, 2010.

Liversidge, H. M.; Speechly, T. \& Hector, M. P. Dental maturation in British children: are Demirjian's standards applicable? Int. J. Paediatr. Dent., 9(4):263-9, 1999.

Maia, M. C.; Martins, Mda. G.; Germano, F. A.; Brandão Neto, J. \& da Silva, C. A. Demirjian's system for estimating the dental age of northeastern Brazilian children. Forensic Sci. Int., 200113):177.e1- .e4, 2010.

Martin-de las Heras, S.; García-Fortea, P.; Ortega, A.; Zodocovich, S. \& Valenzuela, A. Third molar development according to chronological age in populations from Spanish and Magrebian origin. Forensic Sci. Int., 174(1):47-53, 2008.

McKenna, C. J.; James, H.; Taylor, J. A. \& Townsend, G. C. Tooth development standards for South Australia. Aust. Dent. J., 47(3):223-7, 2002.

Meinl, A. The application of dental age estimation methods: comparative validity and problems in practical implementation. Tesis Doctoral. Vienna, University of Vienna, 2007.

Mitchell, J. C.; Roberts, G. J.; Donaldson, A. N. \& Lucas, V. S. Den- tal age assessment (DAA): reference data for British caucasians at the 16 year threshold. Forensic Sci. Int., 189(1-3):19-23, 2009.

Moananui, R. T.; Kieser, J. A.; Herbison, P. \& Liversidge, H. M. Advanced dental maturation in New Zealand Maori and Pacific Island children. Am. J. Hum. Biol., 20(1):43-50, 2008.

Nadler, G. L. Earlier dental maturation: fact or fiction? Angle Orthod., 68(6):535-8, 1998

Nyström, M.; Aine, L.; Peck, L.; Haavikko, K. \& Kataja, M. Dental maturity in Finns and the problem of missing teeth. Acta Odontol. Scand., 58(2):49-56, 2000.

Nyström, M.; Ranta, R.; Kataja, M. \& Silvola, H. Comparisons of dental maturity between the rural community of Kuhmo in northeastern Finland and the city of Helsinki. Community Dent. Oral Epidemiol., 16(4):215-7, 1988.

Olze, A.; Reisinger, W.; Geserick, G. \& Schmeling, A. Age estimation of unaccompanied minors. Part II. Dental aspects. Forensic Sci. Int., 159 (Suppl). 1:S65-7, 2006.

Prieto, J. L. La maduración del tercer molar y el diagnóstico de la edad. Evolución y estado actual de la cuestión. Cuad. Med. Forense, 14(51):11-24, 2008.

Reid, D. J. \& Dean, M. C. Variation in modern human enamel formation times. J. Hum. Evol., 50(3):329-46, 2006.

Tanner, J. M.; Whitehouse, R. H.; Marshall, W. A.; Healy, M. J. R. \& Goldstein, H. Assessment of skeletal maturity and prediction of adult height: TW2 method. London, Academic Press, 1975.

Teivens, A. \& Mörnstad, H. A comparison between dental maturity rate in the Swedish and Korean populations using a modified Demirjian method. J. Forensic Odontostomatol., 19(2):31-5, 2001.

Tunc, E. S. \& Koyuturk, A. E. Dental age assessment using Demirjian's method on northern Turkish children. Turkish children. Forensic Sci. Int., 175(1):23-6, 2008.

Uysal, T.; Sari, Z.; Ramoglu, S. I. \& Basciftci, F. A. Relationships between dental and skeletal maturity in Turkish subjects. Angle Orthod., 74(5):657-64, 2004.

Willems, G. A review of the most commonly used dental age estimation techniques. J. Forensic Odontostomatol., 19(1):9-17, 2001.

Dirección para correspondencia:

Dra. Iris Cadenas Ramos

Unidad de Radiología Maxilofacial, Servicio de Odontología Hospital de Curicó

Curicó

CHILE

Email: irisceleste1977@gmail.com

Recibido : 17-07-2013

Aceptado: 12-11-2014 